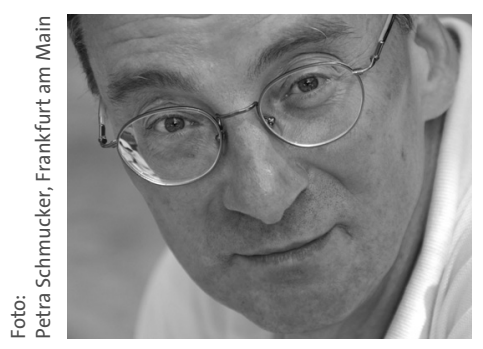

\title{
Offen, aber nicht planlos
}

Wer Visionen habe, sollte zum Arzt gehen, befand einmal Helmut Schmidt. Ein guter Ratschlag gegen allzu weit in die Zukunft reichende Vorstellungen von dem, was kommen wird. Dennoch geht es in Gesellschaft, Politik und auch in der Sozialwirtschaft nicht ohne Pläne und dazu braucht man zumindest einige fundamentale Hinweise auf mögliche Entwicklungen der nächsten Jahre. Und für besonders tiefgreifende und nachhaltige Trends, die gesellschaftliche und technologische Veränderungen betreffen, hat der US-amerikanische Zukunftsforscher John Naisbitt vor dreißig Jahren den Begriff der »Megatrends « geprägt. Attila Nagy, seit über eineinhalb Jahrzehnten beratend in der Sozialwirtschaft tätig, zählt in seinem Einleitungsbeitrag in diesem Heft neun gesellschaftliche Entwicklungen auf und nennt mögliche Auswirkungen auf soziale Leistungserbringer in Deutschland, von der demografischen Entwicklung bis zu technischen Verfahrensinnovationen. Sein Fazit: Soziale Organisationen werden in Zukunft viel stärker als in den letzten Jahrzehnten als innovative soziale Unternehmer gefragt sein.

Der Aufforderung zu sinnvollen Innovationen wollen auch wir uns nicht entziehen. Die vor einem Jahr eingeführten Neuerungen bei der Gestaltung dieser Zeitschrift sind auf ein durchweg positives Echo gestoßen. Nun ziehen wir mit einem konzeptionellen Relaunch nach: Ab dem nächsten Heft erwarten Sie mehr Themen, mehr Kurzbeiträge und mehr umsetzungsorientierte Tipps für den Führungsalltag. Lassen Sie sich überraschen! 